\title{
Adapter le travail à temps partiel aux processus des patients*
}

\author{
Jürg Unger-Köppel \\ Dr méd., membre du Comité central de la FMH, responsable du département Médecine et tarifs hospitaliers
}

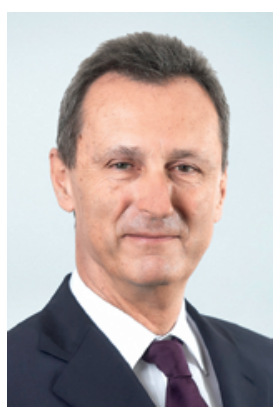

Peu de médecins-chefs peuvent aujourd'hui affirmer en toute âme et conscience pouvoir choisir les meilleurs candidats. Le système a changé à maints égards et le marché de l'emploi a fondamentalement évolué: désormais, ce n'est plus le candidat qui postule mais l'institution. Toute particularité qui rend une institution unique lui procure de gros avantages, car elle est seule à la faire valoir sur le marché. C'est ce qui attire et séduit. Proposer des possibilités de travail à temps partiel attrayantes dans une équipe soudée exerce une force d'attraction formidable sur le marché de l'emploi.

Ce n'est plus le candidat qui postule mais l'institution, dont les chances augmentent grâce aux possibilités de travail à temps partiel.

Les coupler à un bon système de mentorat et à un programme de formation innovant augmente encore les chances sur le marché de l'emploi pour l'institution.

Le travail à temps partiel existe depuis longtemps en médecine. La question ne se pose plus de savoir si nous le voulons ou pas. En revanche, nous devrions trouver ensemble des formes adaptées aux processus des patients et aux collaborateurs. Le temps partiel n'est pas non plus une question de genre, mais une question générationnelle: la génération qui va déterminer l'avenir des hôpitaux préfère souvent travailler à temps partiel, femmes et hommes confondus.

Depuis toujours, on sait que le temps partiel est plus facile à mettre en place dans certaines disciplines: l'anesthésie et la pédopsychiatrie sont le plus souvent évoquées avec, à l'opposé, la néonatologie, la médecine interne ou la chirurgie. La question du travail à temps partiel peut infléchir le choix de la spécialisation en tenant compte de la formation postgraduée et de l'activité de spécialiste envisagée dans une institution ou en cabinet. Il n'est pas rare d'entendre que le temps partiel n'est possible que dans les "petites disciplines», mais c'est oublier que l'hyperspécialisation des "grandes disciplines» réduit aussi l'horizon professionnel en raison d'une très forte focalisation de l'activité. Le temps partiel est tout à fait possible dans les "grandes disciplines» ou celles dans lesquelles il était encore banni il n'y a pas si longtemps. Citons deux exemples: une équipe de médecins cadres en néonatologie veut combiner le travail à temps partiel avec la constance du suivi et du traitement. Elle trouve la solution dans un modèle diachronique de deux postes à $50 \%$ alternant deux semaines à $100 \%$ et deux semaines de temps libre. C'est pour elle le meilleur moyen de s'adapter aux processus des patients. En néphrologie, une équipe en charge d'un centre de dialyse optera pour un système synchronisé au rythme des dialyses, elle s'organisera selon un modèle hebdomadaire classique, dans lequel un poste à 50\% travaille par exemple lundi, mercredi et vendredi matin. Cela montre comment un modèle judicieux peut s'adapter aux processus des patients. Dans un contexte universitaire impliquant des dates de congrès ou des délais de publication à respecter, d'autres options existent comme l'annualisation du temps de travail, qui permet de travailler à un taux élevé pendant une période et de compenser ensuite par plus de temps libre.

Enfin, comment le directeur organise-t-il sa clinique avec des employés à temps partiel? Certainement pas en imposant son modèle. Il est beaucoup plus pertinent de définir le cadre minimal nécessaire pour ceux qui

Le temps partiel existe depuis longtemps en médecine. Trouvons des formes adaptées aux processus des patients et aux collaborateurs.

veulent introduire le temps partiel, dans le but de maintenir la qualité de la prise en charge médicale et de ne pas augmenter le nombre de réunions. Place ensuite à la créativité des intéressés qui ont généralement de l'ambition et sont prêts à prendre des initiatives. Les processus réussiront plus facilement. Le résultat se traduira par une plus forte satisfaction des collaborateurs. C'est une valeur inestimable qui profite directement à la prise en charge des patients. Avec des possibilités de travail à temps partiel dans une équipe satisfaite, une institution dispose d'arguments percutants à faire valoir sur le marché du travail. 